\title{
Control of Nonlinear System with State-Constraints using Barrier Lyapunov Function and Backstepping Method
}

\author{
Joseph R. P. D. Hasibuan ${ }^{1}$, Elfi Yulia ${ }^{2}$, Muhammad Hablul Barri ${ }^{3}$ \\ and Augie Widyotriatmo ${ }^{4, *}$ \\ ${ }^{1,2,3}$ Instrumentation and Control Program, Faculty of Industrial Technology, \\ Institut Teknologi Bandung, Ganesha 10, Bandung 40132, Indonesia. \\ ${ }^{4}$ Instrumentation and Control Research Group, Faculty of Industrial Technology, \\ Institut Teknologi Bandung, Ganesha 10, Bandung 40132, Indonesia. \\ ${ }^{1}$ joseph.hasibuan@gmail.com, ${ }^{2}$ elfiyulia27@gmail.com, \\ ${ }^{3}$ hablul.barri@gmail.com, ${ }^{4}$ augie@tf.itb.ac.id
}

\begin{abstract}
This paper presents the application of Barrier Lyapunov Function (BLF) and backstepping technique to stabilize a nonlinear dynamic system with state-constraints. A barrier function, which limits the region of Lyapunov function candidate, is utilized to design an algorithm that stabilizes each subsystem. Then, a backstepping approach is applied to generate control law that stabilizes the whole system recursively. Using the control law derived from this combination, the origin of state-constrained system is asymptotically stable for all initial conditions within the respective constraints range. For validation, the performance of this proposed control law is demonstrated by simulation, where all of the state trajectories converge to the origin.
\end{abstract}

Keywords: Backstepping, Barrier Lyapunov Function, state-constraints, nonlinear system

\section{Introduction}

Many physical quantities in general, such as electrical signals, air processing, water treatment, robot control, vehicle speed or thermal fluid, have a bounded region of linearity and often constrained operational condition. When controlling the system in linear assumption, just as that boundary is passed, the system may lose its stability. The presence of constraint violation may also degrade the system performance and lead to dangerous situation. This concern has attracted researchers to study control designs on various systems, such as induction motor rotor resistance tracking problem [1], wheeled vehicle robots [2], and rotary drilling system [3].

Methods for modelling and control of nonlinear systems have been widely developed. Stabilization of constrained nonlinear model predictive control (NMPC) [4] [5] is one of the proposed methods. In this method, the control signals are optimized by modifying the reference signals. It is evident that the success of this method depends on the formulation of the optimization and deals with the computational capacity of the given control devices.

The other alternative for nonlinear control designs includes the asymptotic stabilization of nonlinear systems with state-constraints [6]. The Lyapunov stability method is the universal tool that allows investigation of stability of a system and direct design of its control law, which is embedded by appropriately choosing the Lyapunov function [6][7][8]. In the case of the presence of state-constraints, Barrier Lyapunov Function

Received (December 29, 2017), Review Result (February 19, 2018), Accepted (February 20, 2018)

* Corresponding Author 
(BLF) for output tracking is mostly used to stabilize such system as addressed in [8]. The performance of controller designed using BLF has been compared with the Nonlinear Model Predictive Control (NMPC) in [6]. The BLF performed better than the NMPC in computing time and in the aspects of convergence of the states to zero.

Several papers have shown that the control derived using barrier function as a Lyapunov candidate can asymptotically stabilize the nonlinear system with stateconstraints [6] [8]. However, the BLF approach only stabilizes each subsystem in openloop, but not the entirety of the system. The corresponding control signals are also usually the integrator of the barrier-function-based control. Other method for the purpose of such recursive control includes backstepping strategy accompanied by proper signal control reinforcement, which is applied to guarantee small overshoots occurred at the system response [9]. Freeman and Kokotovic in [10] proposed a backstepping method, in which the controller is designed from the known-stable subsystems to stabilize the whole system.

In this paper, we show the design of state-constrained asymptotic stability of nonlinear system using BLF combined with backstepping method. First, the state-constrained nonlinear subsystem is stabilized using the algorithm derived by barrier function as a Lyapunov function candidate. Then, the backstepping method is utilized to obtain the applied control law in stabilizing the whole system.

This paper consists of four sections. The first section is the introduction, which briefly describes the background, objectives and contributions presented from the issues raised. Section 2 presents the problem formulations, which include the nonlinear system under consideration, the properties of barrier function as Lyapunov candidate, the design of subsystem stabilization using BLF, and the application of backstepping method to obtain the control algorithm. Section 3 consists of a simulation of proposed problem showing that the proposed method is applicable. Finally, Section 4 concludes all the results of the proposed method.

\section{Problem Formulation}

In this section, the asymptotic stabilization of a third-order system is discussed. However, the number of states is not limited to be expanded. Let $x=\left[x_{1}(t), x_{2}(t), x_{3}(t)\right]^{T} \in R^{3}$ be the vector of state, $\bar{x}_{1}$ and $\bar{x}_{2}$ be the constraints of $x_{1}$ and $x_{2}$, respectively, where $\left|x_{1}\right|<\bar{x}_{1}$ and $\left|x_{2}\right|<\bar{x}_{2}$, and $u \in R$ is the control input. The function $f(x(t)): D \rightarrow R^{2}$ and $g(x(t)): D \rightarrow R^{2}$ are smooth mapping in the domain $D \subset R^{2}$, where $f(x=0)=0$. The following nonlinear system with stateconstraints is considered:

$\dot{x}_{1}=f\left(x_{1}, x_{2}\right) x_{2}$

$\dot{x}_{2}=g\left(x_{1}, x_{2}\right) x_{3}$

$\dot{x}_{3}=u$.

The system (2.1) represents an open-loop primary systems $x_{1}$ and $x_{2}$, which are nonlinear and constrained, while its control input $u$ is introduced through system $x_{3}$ to stabilize the primary system.

Assumption 1: $g^{-1}\left(x_{1}, x_{2}\right)$ exists for all $x$. 
The control objective is to achieve asymptotic stability of the system states at the origin from the initial value $x_{1}(0) \in \Omega_{1}=\left\{x_{1}(t) \in R:\left|x_{1}\right|<\bar{x}_{1}\right\}$ and $x_{2}(0) \in \Omega_{2}=$ $\left\{x_{2}(t) \in R:\left|x_{2}\right|<\bar{x}_{2}\right\}$, while keeping $x_{1}(t) \in \Omega_{1}$ and $x_{2}(t) \in \Omega_{2}$ for all time. In order to stabilize the system and prevent all state of $x$ from moving out of their constraints, the barrier function as a Lyapunov function candidate, $V_{b}$, is implemented for the individual states $V_{b, i}\left(x_{i}(t)\right):\left(-\bar{x}_{i}, \bar{x}_{i}\right) \rightarrow R^{+}, i=1, . ., n$, which is defined as

$V_{b, i}\left(x_{i}(t)\right)=\frac{1}{2} \ln \frac{\bar{x}_{i}^{2}}{\bar{x}_{i}^{2}-x_{i}^{2}}$,

where $V_{b, i}\left(x_{i}(t)\right)$ is continuous on $\left(-\bar{x}_{i}, \bar{x}_{i}\right)$, positive definite, and $V_{b, i}\left(x_{i}(t) \rightarrow \infty\right.$ as $x_{i}(t) \rightarrow \pm \bar{x}_{i}$. The following Lemma shows the barrier function used in this formulation.

Lemma [6]. Let $y_{i}(t) \in R, i=1, . ., n$. For some constants $\bar{y}_{i}, i=1, \ldots, n$, let $\Omega_{y}=$ $\left\{y(t) \in R:\left|y_{i}(t)\right|<\bar{y}_{i}, i=1, \ldots, n\right\}$. Consider the system

$\dot{y}(t)=h(t, y)$,

where $h: y \rightarrow R$. Let $V_{y, i}:(-\bar{y}, \bar{y}) \rightarrow R^{+}, i=1, \ldots n$ be positive definite function that are continuously differentiable on $\Omega_{y}$. Suppose $V_{y, i}\left(y_{i}\right) \rightarrow \infty$ as $y_{i} \rightarrow \pm \bar{y}_{i}, i=1, . ., n$.

Let $V_{y}(y(t))=\sum_{i=1}^{n} V_{y, i}\left(y_{i}(t)\right)$ and $y(0) \in \Omega_{y}$. If

$\dot{V}_{y}(y(t)) \leq 0$

in the set $\Omega_{y}$, then $y(t) \in \Omega_{y}$ for $t \in[0, \infty]$.

Proof. See [6].

The derivative of barrier function (2.2) with respect to time $t$ can be obtained as:

$\dot{V}_{b, i}\left(x_{i}(t)\right)=\frac{1}{\bar{x}_{i}^{2}-x_{i}^{2}} x_{i} \dot{x}_{i}$,

and by taking

$m\left(x_{i}(t)\right)=\frac{1}{\bar{x}_{i}^{2}-x_{i}^{2}}$,

(2.5) can be simplified with (2.6) into

$\dot{V}_{b}\left(x_{i}(t)\right)=m\left(x_{i}(t)\right) x_{i} \dot{x}_{i}$ 
In this paper, the goal is to stabilize the system as a whole using backstepping method. The method is applied firstly by designing a feedback function $\phi\left(x_{1}, x_{2}\right)$ that stabilizes the systems $x_{1}$ and $x_{2}$. Then, a control input $u$ is derived via backstepping method. The steps are shown as the following:

Step 1. Suppose that the states $x_{1}$ and $x_{2}$ can be stabilized, a smooth state feedback control law $g\left(x_{1}, x_{2}\right) x_{3}=\phi\left(x_{1}, x_{2}\right)$, where $\phi\left(x_{1}, x_{2}\right)$ should be introduced. Then, the subsystems represented in $\dot{x}_{1}$ and $\dot{x}_{2}$ in (2.1) become

$\dot{x}_{1}=f\left(x_{1}, x_{2}\right) x_{2}$

$\dot{x}_{2}=\phi\left(x_{1}, x_{2}\right)$.

Since $x_{1}$ and $x_{2}$ are constrained, the BLF is chosen as

$V_{1}\left(x_{1}, x_{2}\right)=\frac{1}{2}\left(\ln \frac{\bar{x}_{1}^{2}}{\bar{x}_{1}^{2}-x_{1}{ }^{2}}+\ln \frac{\bar{x}_{2}^{2}}{\bar{x}_{2}^{2}-x_{2}{ }^{2}}\right)$

Subsequently, the derivative is as follows:

$$
\begin{aligned}
\dot{V}_{1}\left(x_{1}, x_{2}\right) & =m\left(x_{1}\right) x_{1} \dot{x}_{1}+m\left(x_{2}\right) x_{2} \dot{x}_{2} \\
& =m\left(x_{1}\right) x_{1} f(x) x_{2}+m\left(x_{2}\right) x_{2} \phi\left(x_{1}, x_{2}\right) .
\end{aligned}
$$

This BLF $V_{1}\left(x_{1}, x_{2}\right)$ is known to be smooth and positive definite function, and the derivative $\dot{V}_{1}\left(x_{1}, x_{2}\right)$ satisfies the inequality:

$\dot{V}_{1}\left(x_{1}, x_{2}\right)<-W\left(x_{2}\right)$

where $W$ is a positive function. By choosing

$\phi\left(x_{1}, x_{2}\right)=-\frac{m\left(x_{1}\right)}{m\left(x_{2}\right)} f(x) x_{1}-x_{2}$

as feedback function for backstepping, it can be obtained that

$\dot{V}_{1}\left(x_{1}, x_{2}\right)=-m\left(x_{2}\right) x_{2}^{2} \leq 0$.

The time derivative in (2.12) is negative semi definite, in which $\dot{V}\left(x_{1}, x_{2}\right)=0$ for $x_{2}=0 . x_{1}(t)$ and $x_{2}(t)$ are the solutions of (2.8) starting from $\Omega_{1}$ and $\Omega_{2}$, respectively, and $\dot{V}\left(x_{1}, x_{2}\right) \leq 0$ in both $\Omega_{1}$ and $\Omega_{2}$. Since $\dot{V}\left(x_{1}, x_{2}\right)$ is continuous on the compact set $\Omega_{1}$ and $\Omega_{2}$, it stays in $\Omega_{1}$ and $\Omega_{2}$ as time goes to infinity as in the Lemma. Therefore, $V\left(x_{1}, x_{2}\right)$ has a limit value as $t \rightarrow \infty$. Then, the BarbashinKrasovskii's Theorem is used [11]. Let $Q=\left\{\left[x_{1}(t), x_{2}(t)\right]^{T} \in\left[\Omega_{1}, \Omega_{2}\right]^{T} \mid \dot{V}(x)=0\right\}$ and there exists no solution that can stay identically in $S$ other than the equilibrium 
points of system in (2.8). The set $Q$ can be redefined as $Q=\left\{\left[x_{1}(t), x_{2}(t)\right]^{T} \in\left[\Omega_{1}, \Omega_{2}\right]^{T} \mid x_{2}(t)=0\right\} \quad$ because, if $\dot{V}_{1}\left(x_{1}, x_{2}\right) \equiv 0 \quad$, then $m\left(x_{2}\right) x_{2}{ }^{2}=0$ and, hence, $x_{2}(t)=0$, since $-\bar{x}_{2}<x_{2}(t)<\bar{x}_{2}$.

In consideration to system (2.8), it is also noted that if $x_{2}(t)=0$, then $\dot{x}_{1}(t)=0$, and hence $x_{1}(t)=c$, where $c$ is a constant. This is possible because the presence of function $f(x)$ in (2.8) contributes to the equilibrium point of $x_{1}(t)$ as $c$. In other words, the only solution that can stay identically in $Q$ is the trivial solution $\left[x_{1}(t), x_{2}(t)\right]^{T} \equiv[0, c]^{T}$, where $c$ and 0 are the equilibrium points of $x_{1}(t)$ and $x_{2}(t)$, respectively. Thus, at the equilibrium point of $[0, c]^{T}$ the system is asymptotically stable, and when $f(x)$ contributes to the equilibrium point of $x_{1}(t)$ as 0 , then the origin of $\left(x_{1}, x_{2}\right)$ is asymptotically stable.

Step 2. In the real condition, $g\left(x_{1}, x_{2}\right) x_{3}$ and $\phi\left(x_{1}, x_{2}\right)$ may not be equal. In order to return the expression of (2.8) into (2.1), an equivalent representation of $\dot{x}_{2}$ is derived as

$\dot{x}_{2}=\phi\left(x_{1}, x_{2}\right)+\left[g\left(x_{1}, x_{2}\right) x_{3}-\phi\left(x_{1}, x_{2}\right)\right]$.

Using Assumption 1, $z_{3}$ as a variable form of equivalency can be introduced as

$z_{3}=x_{3}-\frac{\phi\left(x_{1}, x_{2}\right)}{g\left(x_{1}, x_{2}\right)}$

and its derivative with respect to $t$ is as follows:

$$
\begin{aligned}
\dot{z}_{3} & =\dot{x}_{3}-\frac{\partial}{\partial t}\left[\frac{\phi\left(x_{1}, x_{2}\right)}{g\left(x_{1}, x_{2}\right)}\right] \\
& =u-\frac{\partial}{\partial t}\left[\frac{\phi\left(x_{1}, x_{2}\right)}{g\left(x_{1}, x_{2}\right)}\right] .
\end{aligned}
$$

This change of state variables is crucial in stabilizing the entirety of the system with backstepping, such that the system becomes

$\dot{x}_{1}=f\left(x_{1}, x_{2}\right) x_{2}$

$\dot{x}_{2}=\phi\left(x_{1}, x_{2}\right)+g\left(x_{1}, x_{2}\right) z_{3}$

$\dot{z}_{3}=u-\frac{\partial}{\partial t}\left[\frac{\phi\left(x_{1}, x_{2}\right)}{g\left(x_{1}, x_{2}\right)}\right]$.

To fulfil the equivalency role of $z_{3}$ in (2.14), the value of $z_{3}$ has to converge to the origin. Additionally, the constraint for variable $z_{3}$ is not necessarily defined, so that the quadratic Lyapunov function can be used for $z_{3}$, in which such Lyapunov function $V_{2}$ for the system $x_{1}, x_{2}$, and $z_{3}$ is 


$$
V_{2}\left(x_{1}, x_{2}, z_{3}\right)=\frac{1}{2} \ln \frac{\bar{x}_{1}^{2}}{\bar{x}_{1}^{2}-x_{1}^{2}}+\frac{1}{2} \ln \frac{\bar{x}_{2}^{2}}{\bar{x}_{2}^{2}-x_{2}^{2}}+\frac{1}{2} z_{3}^{2},
$$

where its derivative is defined as

$$
\begin{aligned}
\dot{V}_{2}\left(x_{1}, x_{2}, z_{3}\right) & =m\left(x_{1}\right) x_{1} \dot{x}_{1}+m\left(x_{2}\right) x_{2} \dot{x}_{2}+z_{3} \dot{z}_{3} \\
& =m\left(x_{1}\right) x_{1} f\left(x_{1}, x_{2}\right) x_{2}+m\left(x_{2}\right) x_{2} \phi\left(x_{1}, x_{2}\right)+z_{3}\left[m\left(x_{2}\right) x_{2} g\left(x_{1}, x_{2}\right)+\dot{z}_{3}\right] .
\end{aligned}
$$

By utilizing $\phi\left(x_{1}, x_{2}\right)$ in (2.11) and $\dot{z}_{3}$ in (2.16), (2.18) is reduced into

$$
\begin{aligned}
\dot{V}_{2}\left(x_{1}, x_{2}, z_{3}\right) & =-m\left(x_{2}\right) \cdot x_{2}{ }^{2}+z_{3}\left[m\left(x_{2}\right) x_{2} g\left(x_{1}, x_{2}\right)+\dot{z}_{3}\right] \\
= & -m\left(x_{2}\right) x_{2}{ }^{2}+z_{3}\left[m\left(x_{2}\right) x_{2} g\left(x_{1}, x_{2}\right)+u-\frac{\partial}{\partial t}\left(\frac{\phi\left(x_{1}, x_{2}\right)}{g\left(x_{1}, x_{2}\right)}\right)\right] .
\end{aligned}
$$

Taking

$u=\frac{\partial}{\partial t}\left(\frac{\phi\left(x_{1}, x_{2}\right)}{g\left(x_{1}, x_{2}\right)}\right)-m\left(x_{2}\right) x_{2 .} g\left(x_{1}, x_{2}\right)-z_{3}$

yields the derivative of $V_{2}$ as follows:

$\dot{V}_{2}\left(x_{1}, x_{2}, z_{3}\right)=-m\left(x_{2}\right) x_{2}^{2}-z_{3}^{2}$,

which concludes that $z_{3}$ goes to zero as well. This shows that the system (2.16) becomes equivalent with (2.8). Thus, the control law derived by using BLF and backsteping methods asymptotically stabilizes the system at $\left[x_{1}(t), x_{2}(t), x_{3}(t)\right]^{T} \equiv[0, c, 0]^{T}$, or at the origin when $f(x)$ contributes to $c=0$. The result is concluded in the following theorem:

Theorem. Consider the system (2.1) with Assumption 1, state-constraints $\left|x_{1}\right|<\bar{x}_{1}$, $\left|x_{2}\right|<\bar{x}_{2}$, and the initial values $x_{1}(0) \in \Omega_{1}=\left\{x_{1}(t) \in R:\left|x_{1}\right|<\bar{x}_{1}\right\}, x_{2}(0) \in \Omega_{2}=\left\{x_{2}(t) \in\right.$ $\left.R:\left|x_{2}\right|<\bar{x}_{2}\right\}$. Using the control law (2.20), the origin of $\left[x_{1}(t), x_{2}(t), x_{3}(t)\right]^{T} \equiv[0,0,0]^{T}$ is asymptotically stable.

\section{Simulation Result}

In this section, a numerical study through simulation of a single-input third-order system is provided. A case of nonlinear system being considered is as the following:

$$
\begin{aligned}
& \dot{x}_{1}=x_{1} x_{2} \\
& \dot{x}_{2}=\frac{x_{3}}{2+\sin \left(x_{1}\right)} \\
& \dot{x}_{3}=u .
\end{aligned}
$$


By substituting $\frac{x_{3}}{2+\sin \left(x_{1}\right)}$ with $\phi\left(x_{1}, x_{2}\right)$ as state feedback control law, backstepping is performed. Then, a barrier Lyapunov function in (2.2) is used as the Lyapunov function candidate for system $x_{1}$ and $x_{2}$. To stabilize the system, the time derivative of Lyapunov function is considered:

$$
\dot{V}_{b}\left(x_{1}, x_{2}\right)=m\left(x_{1}\right) x_{1}^{2} x_{2}+m\left(x_{2}\right) x_{2} \phi\left(x_{1}, x_{2}\right) .
$$

By designing feedback function $\phi\left(x_{1}, x_{2}\right)$ as

$$
\phi\left(x_{1}, x_{2}\right)=-\frac{m\left(x_{1}\right)}{m\left(x_{2}\right)} x_{1}^{2}-x_{2},
$$

the time derivative of $V_{b}$ becomes

$$
\begin{aligned}
\dot{V}_{b}\left(x_{1}, x_{2}\right) & =-m\left(x_{2}\right) x_{2}{ }^{2} \\
& \leq 0,
\end{aligned}
$$

in the set of $\left|x_{1}\right|<\bar{x}_{1}$ and $\left|x_{2}\right|<\bar{x}_{2}$.

Then, as in (2.13), $z_{3}$ is defined as

$$
z_{3}=x_{3}-\phi\left(x_{1}, x_{2}\right) \sin \left(x_{1}\right),
$$

so that the time derivative of (2.14) can be given by the following equation:

$$
\begin{aligned}
\dot{z}_{3} & =\dot{x}_{3}-\frac{\partial}{\partial t}\left[\phi\left(x_{1}, x_{2}\right) \sin \left(x_{1}\right)\right] \\
& =u-\left\{\frac{\partial}{\partial x_{1}}\left[\phi\left(x_{1}, x_{2}\right) \sin \left(x_{1}\right)\right] \dot{x}_{1}+\frac{\partial}{\partial x_{2}}\left[\phi\left(x_{1}, x_{2}\right) \sin \left(x_{1}\right)\right] \dot{x}_{2}\right\}
\end{aligned}
$$

while the expression of $\dot{x}_{1}$ and $\dot{x}_{2}$ for (3.6) is taken from the transformed system as the following:

$$
\begin{aligned}
& \dot{x}_{1}=x_{1} x_{2} \\
& \dot{x}_{2}=\phi\left(x_{1}, x_{2}\right)+\frac{x_{3}}{2+\sin \left(x_{1}\right)} \cdot z_{3} \\
& \dot{z}_{3}=u-\left\{\frac{\partial}{\partial x_{1}}\left[\phi\left(x_{1}, x_{2}\right) \sin \left(x_{1}\right)\right] \dot{x}_{1}+\frac{\partial}{\partial x_{2}}\left[\phi\left(x_{1}, x_{2}\right) \sin \left(x_{1}\right)\right] \dot{x}_{2}\right\} .
\end{aligned}
$$

By using the Lyapunov function in (2.17) and the differential equation (3.7), let the input function be designed as

$$
u=\left\{\frac{\partial}{\partial x_{1}}\left[\phi\left(x_{1}, x_{2}\right) \sin \left(x_{1}\right)\right] \dot{x}_{1}+\frac{\partial}{\partial x_{2}}\left[\phi\left(x_{1}, x_{2}\right) \sin \left(x_{1}\right)\right] \dot{x}_{2}\right\}-m\left(x_{2}\right) x_{2 .} g(x)-z_{3},
$$

which yields the Lyapunov function's time derivative as in (2.21).

The performance of the designed control law for the system in (3.1) is then observed in the phase portrait in Figure 1. The constraints $\bar{x}_{1}$ and $\bar{x}_{2}$ are set to be 1.00. The trajectories of $x_{1}(t)$ and $x_{2}(t)$ converge to the origin without violating the constraints because the system (3.1) has $f(x)=x_{1}$, which provides the system with an equilibrium point $x_{1}=0$. Hence, the system is asymptotically stable at the origin. Figure 2 shows the trajectories of $x_{1}(t)$ and $x_{2}(t)$ of the system (3.1) with respect to $t$ 
when the designed control input (3.8) is used. While $\bar{x}_{1}$ and $\bar{x}_{2}$ are set to be 1.00 , the initial conditions are chosen to be near the constraints, namely $x_{1}(0)=x_{2}(0)=0.95$ and $x_{3}(t)=0.00$. From the Figure 2, the BLF prevents both variables $x_{1}(t)$ and $x_{2}(t)$ to violate the individual constraints. The control input is shown in Figure 3.

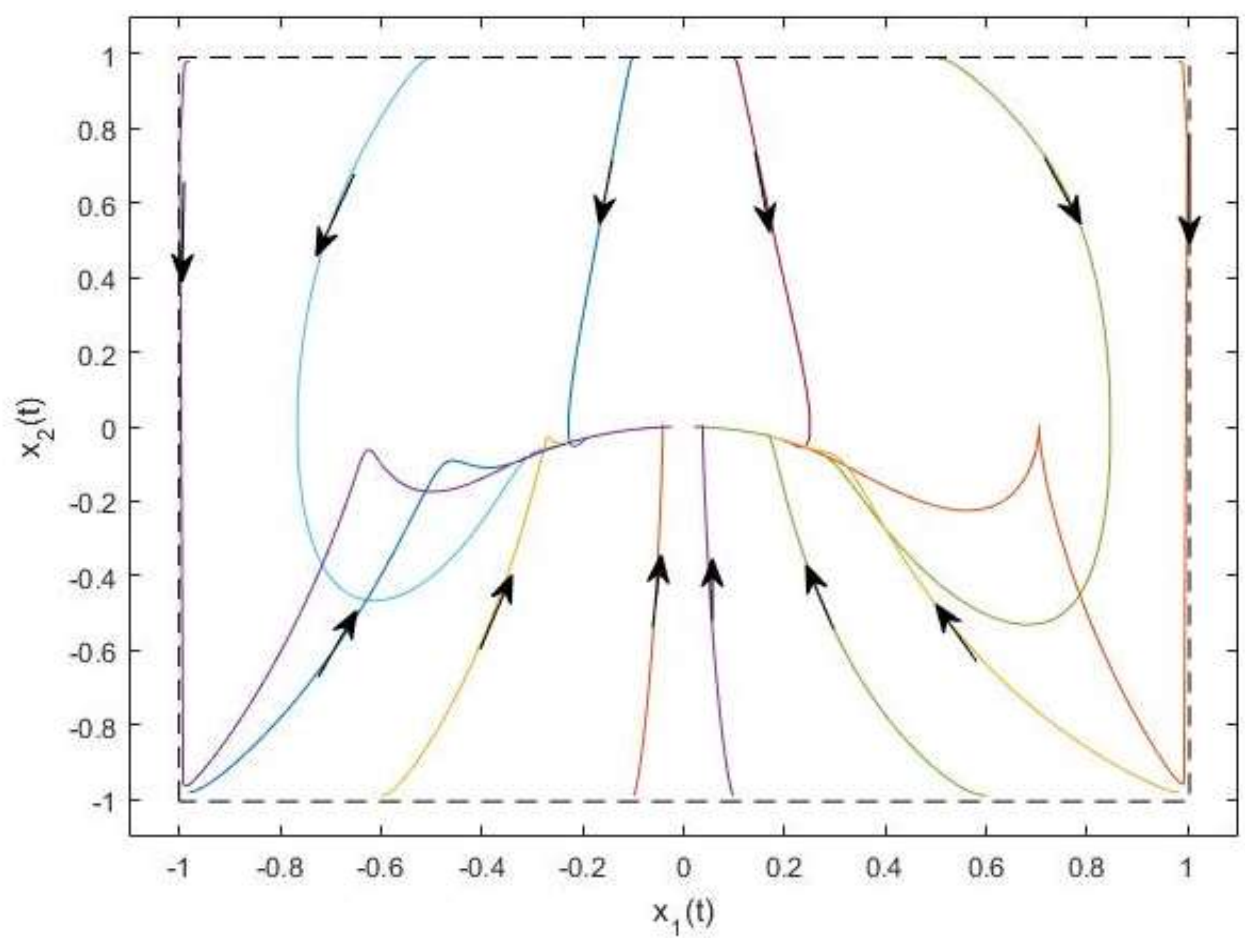

Figure 1. Phase Portraits of States $x_{1}(t)$ and $x_{2}(t)$ of the System (3.1) using Control Law (3.8) with Constraints $\bar{x}_{1}=\bar{x}_{2}=1.00$

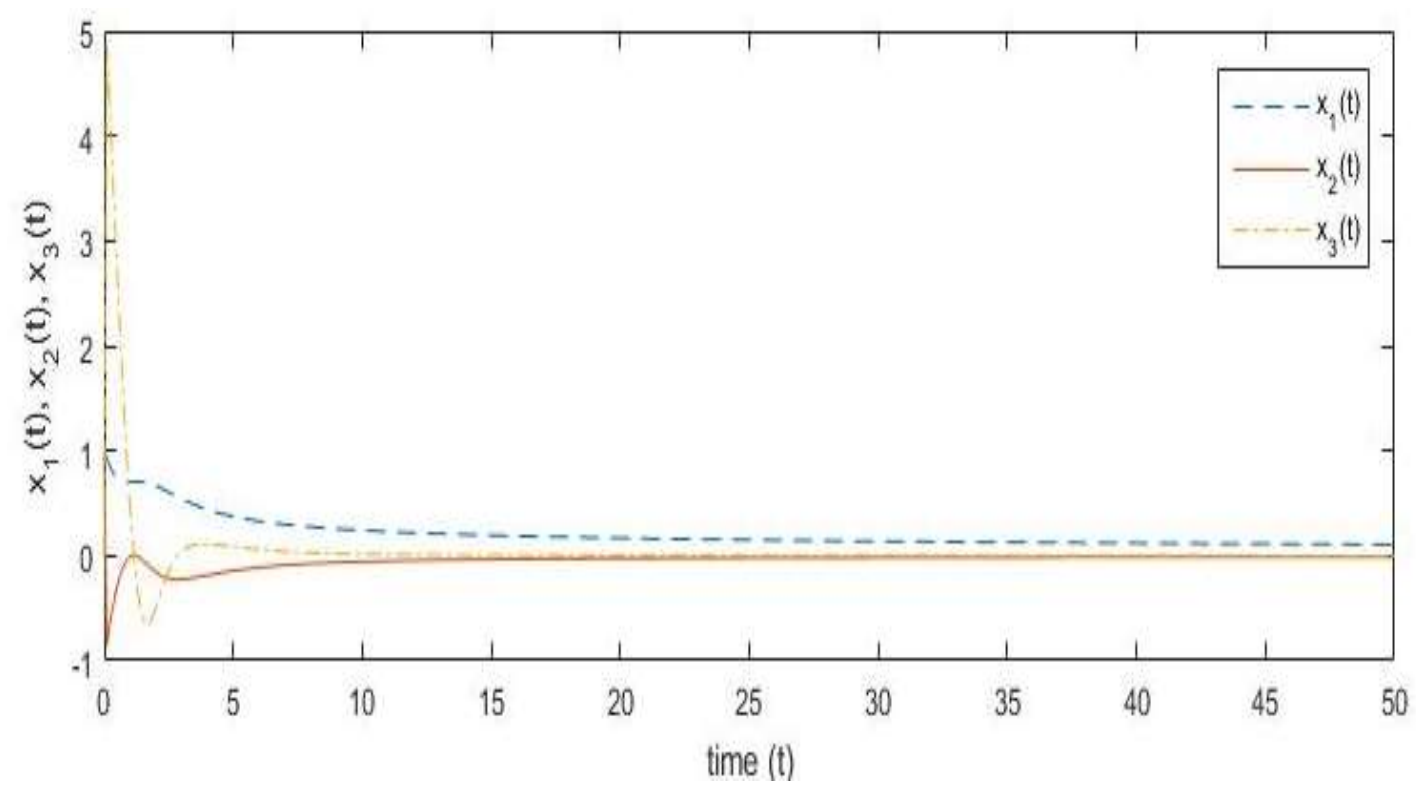

Figure 2. Trajectories of $x_{1}(t)$ and $x_{2}(t)$ of the System (3.1) using Control

Law (3.8) with Initial Conditions $x_{1}(0)=x_{2}(0)=0.95$ and $x_{3}(0)=0$, the

Constraints $\bar{x}_{1}=\bar{x}_{2}=1.00$ 


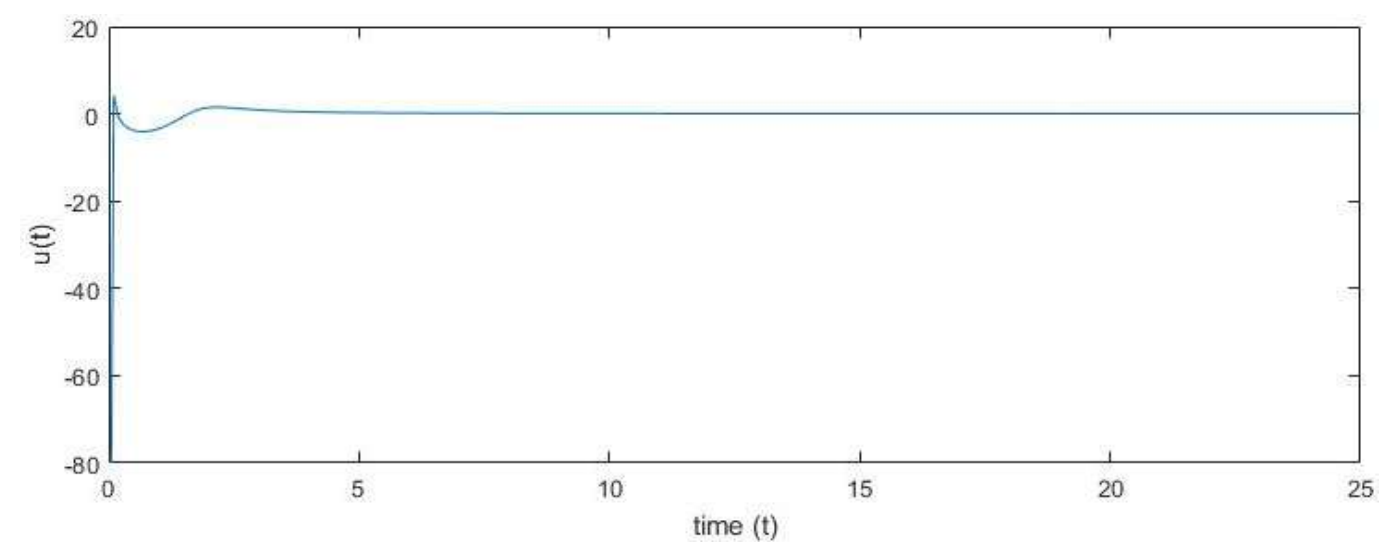

Figure 3. Control Input Used in Simulation Depicted in Figure 2

\section{Conclusion}

This paper has addressed the problem of stability in state-constrained nonlinear dynamic system by designing the control law using the barrier function as a Lyapunov function candidate and the backstepping method. The barrier-function-based control has been utilized to stabilize each of the subsystems, in which the states have been constrained within certain values. Then, the backstepping method has also been applied to obtain the control law for the whole system from the stabilized subsystem. The combination of the barrier-function-based method and the backstepping method has provided the direct control design for a constrained nonlinear system recursively. The simulation has, thus, proved that the states have been in asymptotically stable condition at the origin while not violating their respective given constraints.

\section{Acknowledgement}

This work was supported by a fundamental research grant from KEMENRISTEKDIKTI (Indonesian Ministry of Research, Technology and Higher Education).

\section{References}

[1] C. B. Regaya, F. Farhani, A. Zaafouri and A. Chaari, "A Novel Adaptive Control Method for Induction Motor Based on Backstepping Approach using dSpace DS 1104 Control Board", Mechanical System and Signal Processing, vol. 100, (2017), pp. 466-481.

[2] A. Widyotriatmo and K.-S. Hong, "Switching Algorithm for Robust Configuration Control of a Wheeled Vehicle", Control Engineering Practice, vol. 20, no. 3, (2012), pp. 315-325.

[3] R. Mlayeh, S. Toumi, and L. Beji, "Backstepping Boundary Observer Based-Control for Hyperbolic PDE in Rotary Drilling System", Applied Mathematics and Computation, vol. 322, (2017), pp. 66-78.

[4] D. Q. Mayne and J. B. Rawlings, C. V. Rao, and P. O. M. Scokaert, "Constrained Model Predictive Control: Stability and Optimality”, Automatica, vol. 36, no. 6, (2000), pp. 789-814.

[5] P. Mhaskar, N. H. El-Farra, and P. D. Christofides, "Stabilization of Nonlinear System with State and Control Constraints using Lyapunov-based Predictive Control", American Control Coference, Oregon, USA, (2005) June 8-10.

[6] A. Widyotriatmo and K.-S. Hong, "Asymptotic Stabilization of Nonlinear System with State Constraints", International Journal of Applied Mathematics and Statistics, vol. 53, No. 3, (2015), pp. 1023.

[7] A. Widyotriatmo and K.-S. Hong, "Navigation Function-Based Control of Multiple Wheeled Vehicles", IEEE Transaction on Industrial Electronics, vol. 58, no. 5, (2011), pp 1896-1906.

[8] K. B. Ngo, R. Mahony and Z.-P. Jiang, "Integrator Backstepping Functions for Systems with Multiple State Constraints", Proceedings of the 44th IEEE Conference on Decision and Control, Seville, Spain (2005) December 15-15.

[9] M. Krstic, A. A. Siranosian, A. Smyshlyaev and M. Bement, "Backstepping Boundary Controllers and Observers for the Slender Timoshenko Beam: Part II---Stability and Simulations", 45 $5^{\text {th }}$ IEEE Conference on Decision and Control, California, USA, (2006) December 13-15. 
[10] R. A. Freeman and P. Kokotovic, "Robust Nonlinear Control Design: State-space and Lyapunov Technique", Modern Birkhäuser Classics, Boston, (1996).

[11] H. K. Khalil, "Nonlinear Systems, $3^{\text {rd }}$ Edition", Prentice Hall, New Jersey, (2002), pp. 128-129.

\section{Authors}

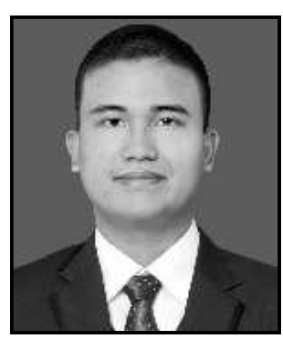

Joseph Ramos Pararat Diogoamas Hasibuan, received his B. Eng in Engineering Physics from Institut Teknologi Bandung, Indonesia in 2015. He is currently pursuing a Master Degree in Instrumentation and Control Engineering at the Faculty of Industrial Technology at Institut Teknologi Bandung, Indonesia. His research interests include nonlinear system, multi-agent control, and plantwide control.

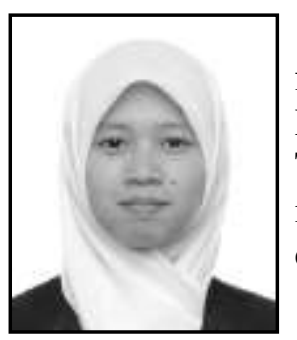

Elfi Yulia, received her bachelor degree in Engineering Physics from Institut Teknologi Bandung. She is currently pursuing a Master Degree in Instrumentation and Control at the Faculty of Industrial Technology, Institut Teknologi Bandung. Her research interests include design of piezoelectric speed bump as energy system and control engineering.

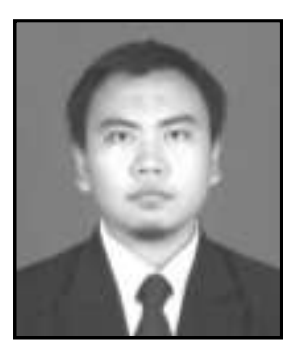

Muhammad Hablul Barri, received his B.Eng in Electrical Engineering from Institut Teknologi Sepuluh Nopember, Indonesia. $\mathrm{He}$ is currently pursuing a Master Degree in Instrumentation and Control at Faculty of Industrial Technology, Institut Teknologi Bandung, Indonesia. He is a LPDP scholarship awardee. His research interests include robotics, nonlinear control, biomedical instrumentation, and image processing and analysis.

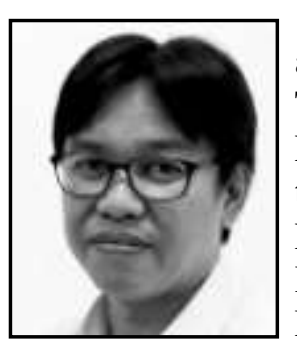

Augie Widyotriatmo, received his B. Eng. in Engineering Physics and M. Eng. in Instrumentation and Control from the Institut Teknologi Bandung, Indonesia and Ph.D. degree in Mechanical Engineering from Pusan National University, South Korea. He joined the Faculty of Industrial Technology as a faculty member for Engineering Physics program in the Institut Teknologi Bandung Indonesia in 2010. He was a visiting scholar in the University of Melbourne, Australia, in November-December 2016. His research interests include robotics, nonlinear control, energy optimization, control, and automation, biomedical instrumentation, automation system, and power system control. Dr. Widyotriatmo is the chair of the IEEE Indonesia Control Systems and Robotics and Automation Joint Chapter Societies for the period of 2017-2018. He is an associate editor in the Journal of Measurements, Electronics, and Systems (JMECS). He was the Guest Editor in the Internetworking Indonesia Journal in 2017. He was the General Chairs of the 3rd International Conference on Instrumentation, Control, and Automation in 2013 and the IEEE Conference on Technology, Informatics, Management, Engineering, and Environment in 2013. 